\title{
Crystallization of $\mathrm{L}$-alanine in the presence of additives on a circular PMMA platform designed for metal-assisted and microwave-accelerated evaporative crystallization $\dagger$
}

\author{
Anginelle M. Alabanza ${ }^{b}$, Muzaffer Mohammed ${ }^{a}$, and Kadir Aslan ${ }^{a}$ \\ Kadir Aslan: Kadir.Aslan@morgan.edu \\ aMorgan State University, Department of Chemistry, 1700 East Cold Spring Lane, Baltimore, MD, \\ 21251, USA \\ bThe College of New Jersey, Department of Chemistry, 2000 Pennington Road, Ewing, NJ, \\ 08628, USA
}

\section{Abstract}

Crystallization of $\mathrm{L}_{\mathrm{L}}$ alanine in the presence of $\mathrm{L}_{\mathrm{-}} \mathrm{valine}$ and $\mathrm{L}$-tryptophan additives on a circular poly(methyl) methacrylate (PMMA) platform designed for Metal-Assisted and MicrowaveAccelerated Evaporative Crystallization (MA-MAEC) technique was investigated. Theoretical simulations predicted homogeneous temperature and electric field distributions across the circular PMMA platforms during microwave heating. Crystallization of L-alanine with and without additives on the blank and silver nanoparticle films (SNFs) modified sides of the circular PMMA platform occurred within 32-50 min using MA-MAEC technique, while the identical solutions crystallized within 161-194 min at room temperature. Optical microscopy studies revealed that $\mathrm{L}^{-}$ alanine crystals without additives were found to be smaller in size and had several well-developed faces, whereas L-alanine crystals grown with additives appeared to be larger and had only one dominant highly-developed face. Raman spectroscopy and powder X-ray diffraction (XRD) measurements showed that all $\mathrm{L}$-alanine crystals had identical peaks, despite the morphological differences between the $\mathrm{L}_{\mathrm{L}}$-alanine crystals with and without additives observed by optical microscope images.

\section{Introduction}

Evaporative crystallization is one of the important industrial processes used for the purification of molecules of interest in the pharmaceutical industry. With the increasing number of newly synthesized drugs and molecules, it is critical that these materials are purified with a good control over crystal size and in a timely manner for the characterization step. In response to the strong demand for more efficient crystallization techniques and platforms, new and innovative methods, including polarized laser light irradiation in solution, ${ }^{1}$ and engineered surfaces such as nanoscale cylindrical pores, ${ }^{2}$ platforms for microdroplet solvent evaporation and self-assembled monolayers (SAMs) of alkane thiols on patterned surfaces ${ }^{3-5}$ through solvent evaporation are being developed. Most notably, Kenis group recently reported a microfluidic platform comprised of 48 wells to screen for pharmaceutical salts and polymorphs of active pharmaceutical ingredients (API) through antisolvent crystallization. ${ }^{6,7}$ Despite the significant advances made in all these publications,

\footnotetext{
${ }^{\dagger}$ Electronic supplementary information (ESI) available. See DOI: 10.1039/c2ce26363g

(C) The Royal Society of Chemistry 2012

Correspondence to: Kadir Aslan, Kadir. Aslanemorgan. edu.
} 
evaporative crystallization based on the traditional room temperature crystallization method still takes hours to days to occur in laboratory bench scale. ${ }^{8-10}$

The Aslan Research Group has recently developed a new evaporative crystallization method, called MA-MAEC, in which plasmonic nanostructures (i.e., SNFs) are employed along with microwave heating in order to accelerate the crystal growth process without altering the physical properties of the crystal. Using the MA-MAEC technique, crystallization of amino acids and drug compounds (from $<1 \mathrm{ml}$ samples) can be carried out "on-demand" within seconds. To date, the Aslan Research Group has demonstrated the use of MA-MAEC technique for the rapid crystallization of glycine, L-arginine acetate, L-alanine

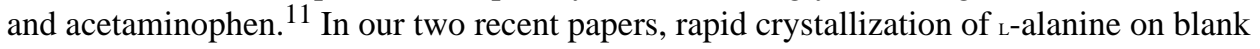
glass slides and SNFs and surface engineered SNFs using MA-MAEC was demonstrated. ${ }^{8,12}$ These studies showed that the use of MA-MAEC can reduce total crystallization time from hours to seconds while improving the size, organization and quality of ${ }_{\text {L-alanine crystals produced. }}{ }^{8}$ The success of MA-MAEC was partially attributed to the presence of silver nanostructures, which serve as selective nucleation sites for the growth of L-alanine crystals. Furthermore, the incorporation of microwave heating introduces a thermal gradient in which SNFs remain at room temperature while the rest of the system is heated. ${ }^{9}$ During microwave heating, in an attempt to accomplish a thermal equilibrium amino acid molecules move from their initial position in the warmer solution to the cooler SNFs surface. As microwave heating continues, the remaining amino acid molecules are transferred from the solution, and assemble onto SNFs, where L-alanine crystals grow (i.e., thermal gradient induced mass transfer). ${ }^{9}$

Another interesting component of crystallization of amino acids is the incorporation of additives (i.e., other amino acids) into an amino acid solution, which results in morphological changes in crystal structure of the amino acids. ${ }^{13-15}$ It is thought that these additives selectively absorb onto specific faces of the crystals, modifying the growth of other faces and therefore resulting in alterations of their morphologies. ${ }^{15,16}$ The use of additives in crystallization is of high significance to the pharmaceutical industry due to their potential ability to help synthesize drugs in crystal form with particular intended physiochemical properties. ${ }^{16,17}$ For example, Lahav and Leiserowitz ${ }^{16}$ described the relationship between additive structure and crystal morphology with $(S)$-asparagine- $\mathrm{H}_{2} \mathrm{O}$. Without any additives, the crystal expresses little of the (010) face, which one $\mathrm{O}-\mathrm{H}$ bond per water molecule is associated with, according to the crystal's packing arrangement. When methanol is added as a tailor-made solvent-additive, methanol molecules are able to attach to these areas. As a result, methanol's methyl groups stick out of both (010) faces and inhibit crystal grown in the $b$-direction. This creates two large $(010)$ faces which are seen in $(S)$ asparagine- $\mathrm{H}_{2} \mathrm{O}$ crystals doped with methanol additive, but are not seen in the pure form of the crystal. ${ }^{16}$

In our previous publications on MA-MAEC, $, 9,9,18$ rectangular glass microscope slides (length $\times$ width $=7.5 \times 2.5 \mathrm{~cm}^{2}$ ) were used as the crystallization platforms due to their wide availability and amenability to the facile attachment of SNFs. However, the sharp corners of glass slides result in the reflection of electric field of the microwaves and subsequently, in a heterogeneous heated surface on which crystal growth occurs, which limited the use of glass slides in multiplexed crystallization of amino acids from samples with small volumes. The use of rectangular glass microscope slides in MA-MAEC can produce imperfect crystals due to varying surface temperatures and overheating across the glass microscope slide. One can alleviate the heterogeneous heating issue by using a crystallization platform without sharp edges that can maintain homogenous heating in a microwave oven. 
In this work, we introduce a new crystallization platform constructed from circular poly(methyl methacrylate) (PMMA) and modified with SNFs and 16-well silicon isolators. The new circular PMMA crystallization platforms are designed to reduce the effects of temperature variations caused by microwaves by allowing the electric field component of the microwaves to flow around the circular surface rather than reflect them. It is also important to note that the size of the circular PMMA crystallization platforms is smaller (5 $\mathrm{cm}$ diameter) than the wavelength of the microwaves $(\sim 12.2 \mathrm{~cm})$ employed, which reduces the interaction of the PMMA platforms with the heterogeneous electric field component of a conventional microwave oven. The homogenous heating of circular PMMA crystallization platforms also affords for high throughput small-scale production of amino acid crystals. PMMA is a routinely synthesized vinyl polymer that is widely used in areas such as bioassays and medical technology. ${ }^{19}$ Its stable plasticity, and homogeneity over its entire surface $^{20}$ suggest that PMMA is very suitable for high throughput small-scale crystal production. Subsequently, PMMA surfaces were activated by plasma cleaning and conventional surface modification for the attachment of SNFs and the growth of $\mathrm{L}_{\mathrm{L}}$ alanine. The effect of additives (L-valine and $\mathrm{L}_{\mathrm{L}}$ tryptophan) on $\mathrm{L}$-alanine crystallization on a SNFscoated circular PMMA platform was also investigated. With the use of MA-MAEC, it is found that the use of $\mathrm{L}_{\mathrm{-}}$-valine and $\mathrm{L}_{\mathrm{L}}$ tryptophan (isoelectric point of $\mathrm{L}_{\mathrm{L}}$-valine $=5.96$, $\mathrm{pI}$ of $\mathrm{L}_{\mathrm{L}}$ tryptophan $=5.89$ are close to that of $\mathrm{L}_{\mathrm{L}}$-alanine, isoelectric point $=6.11$ ) as additives resulted in an increase the size and quality of ${ }_{\mathrm{L}}$-alanine crystals. The complete evaporation of the solvent took significantly longer than those observed on glass microscope slides: 32-50 min and 161-194 min using MA-MAEC technique and room temperature evaporation (control experiment), respectively. The longer evaporation times was attributed to the better control of temperature and electric field distributions across the circular PMMA platforms and the use of silicone isolators that absorb excess microwave heating. Characterization of the $\mathrm{L}^{-}$ alanine crystals was carried out using optical microscopy, Raman spectroscopy and powder XRD.

\section{Materials and methods}

\section{Materials}

PMMA disks (diameter $=5 \mathrm{~cm}$ ) were obtained from McMaster-Carr (IL, USA). Silicone isolators with 12 wells (each well holds $30 \mu \mathrm{L}$ of water) and silver target ( $57 \mathrm{~mm}$ diameter) were purchased from Electron Microscopy Sciences. L-alanine, L-valine, and L-tryptophan were purchased from Sigma-Aldrich and were used without further purification.

\section{Methods}

Preparation of circular PMMA crystallization platform-PMMA disks were plasma cleaned (Harrick Plasma PDC-32G) before use. One half of the disk was then immediately coated with $1 \mathrm{~nm}$ thick SNFs using a sputter coater (EMS 150R S, Electron Microscopy Sciences, Hatfield, PA, USA), while the other half was left uncoated. Silicone isolators with 12 wells were applied directly to both silver-coated and uncoated disk surfaces, and used as is.

Preparation of solutions-A fresh $2.0 \mathrm{M}$ solution was prepared from $\mathrm{L}$-alanine and deionized water (Millipore, Direct-Q UV, $18.2 \mathrm{ohm} \mathrm{m}$ ) and maintained at $75^{\circ} \mathrm{C}$ on a Corning magnetic stirrer/heater. An appropriate amount of $0.5 \%(\mathrm{w} / \mathrm{W})$ of $\mathrm{L}_{\mathrm{L}}$ tryptophan or $\mathrm{L}_{\mathrm{L}}$ valine was added to the solution after all L-alanine had completely dissolved. Solution was observed for transparency and any undissolved particles before beginning crystallization process. To prevent the possibility of alterations to its solubility curve due to continuous heating, a new solution was prepared each day. 
Crystallization of L-alanine-Crystallization of ${ }_{\mathrm{L}}$-alanine with and without additives was performed on blank and SNFs-coated circular PMMA platforms at room temperature and using the MA-MAEC technique, as previously described. ${ }^{8}$ A $20 \mu \mathrm{L}$ of solutions was placed into the wells of the silicon isolators on the circular PMMA platforms. Crystals grown at room temperature were set aside and remained undisturbed for the duration of the crystallization period. Crystals grown using microwave heating were done in a $0.9 \mathrm{cu}$. ft, $900 \mathrm{~W}$ conventional microwave oven (Frigidaire Model No. FCM09Z03KB) at power level 1 , or $10 \%$ power. Circular PMMA platforms were placed in the center of the microwave in all experiments, to ensure homogenous heating throughout the cycle.

Characterization of L-alanine crystals-Optical images were taken using a Swift Digital M10L monocular microscope that was connected to a PC equipped with an image processing software provided by the vendor. Crystals were characterized by Raman spectroscopy (i-Raman, BW\&Tek, Inc.) and powder X-ray diffraction (XRD).

Theoretical simulations-Simulations of microwave heating of circular PMMA platforms were run using the RF module of the commercially available software COMSOL Multiphysics $^{\mathrm{TM}}$, Version 4.3 (COMSOL, Inc., Burlington, MA, USA).

Characterization of SNFs-SNFs were characterized a Varian Cary 50 UV-Vis spectrophotometer and atomic force microscopy. Real color photos of blank PMMA and SNFs-coated circular PMMA platforms were taken using a 5 MP digital camera.

\section{Results and discussion}

Since circular SNFs-coated PMMA platforms (i.e., circular PMMA platforms) were designed as a crystallization platform for use in MA-MAEC, it is important to investigate the heating characteristics in a conventional microwave using theoretical calculations. Circular PMMA platforms (has two sides: blank and SNFs-coated) are thought to afford for homogenous heating throughout the disk over the entire heating cycle due to their small size and circular shape. The duty cycle of the conventional microwave oven used in this study is $\sim 3 \mathrm{~s}$ (Fig. S1, ESI $\dagger$ ): using power level 1 setting the full microwave power is on for $3 \mathrm{~s}$, which is followed by $\sim 30 \mathrm{~s}$ of period of no microwave power. It is expected that $3 \mathrm{~s}$ of microwave heating increases the temperature of the solution after which is cooled during the subsequent 30 s periods. Given these observations, theoretical calculations were carried out for $3 \mathrm{~s}$ of microwave heating. Fig. 1 shows the temperature variation and electric field ( $z$ component) distribution after $3 \mathrm{~s}$ of microwave heating in a conventional microwave oven with a circular PMMA platform using theoretical calculations. The temperature variation between all 16 wells of the PMMA platform was predicted to differ by $<1{ }^{\circ} \mathrm{C}$ after $3 \mathrm{~s}$ periods of microwave heating. Subsequently, microwave heating of a solution at an initial temperature of $75^{\circ} \mathrm{C}$ for $3 \mathrm{~s}$ is predicted to reach a maximum temperature is $\sim 77.5^{\circ} \mathrm{C}$. The minimal variation in temperature due to microwave heating (heating-cooling cycles) implies that PMMA platforms are appropriate for MA-MAEC technique. ${ }^{11}$ Fig. 1 also displays the predicted intensity of the $z$-component of the electric field. As expected from a conventional oven, electric field is heterogeneous throughout the microwave cavity and the rotation of the circular PMMA platforms ensures that homogenous microwave heating can be attained over the duration of crystallization experiments. It is important to note that microwave power level 1 was determined to provide optimum conditions for the MA-MAEC technique, based on the observations that at other power levels for the conventional microwave oven the $\mathrm{L}_{-}$ alanine solutions evaporate rapidly without the growth of high quality L-alanine crystals (for the sake of brevity, the large amount of "negative" data supporting this claim are not shown). 
As discussed previously, the success of the MA-MAEC technique depends on the use of SNFs (as well as other plasmon-supporting nanostructures such as gold, copper, aluminum, iron, tin, zinc). ${ }^{21}$ In this regard, SNFs were deposited onto the selective regions of the blank PMMA disks using a mask. Subsequently, silicon isolators were applied to the circular PMMA platforms where the location of the wells matched the location of SNFs as shown in Fig. 2 inset. The real-color photograph of the circular PMMA platforms (Fig. 2 inset) show that the SNFs were deposited as semi-continuous film in a homogeneous manner. To corroborate this observation, SNFs were characterized using UV-Vis spectroscopy as shown in Fig. 2. As compared to the low absorbance values of blank PMMA disk, the higher absorbance values at longer wavelengths of circular PMMA platform indicates the presence of silver on the disk. Furthermore, the broadening of the absorption spectrum at 550 to 800 $\mathrm{nm}$ implies that a semi-continuous film of silver was deposited. This observation was further verified by an AFM image of the circular PMMA platforms (Fig. S2, ESI $\dagger$ ).

In order to demonstrate the use of circular PMMA platforms in MA-MAEC based crystallization and to serve as a control experiment, the crystallization of $\mathrm{L}_{\mathrm{L}}$-alanine without additives was carried out initially. The results of these experiments are summarized in Table 1, and Fig. S3-S7 and S28 (ESI $\dagger$ ). The average complete evaporation of $\mathrm{L}_{\mathrm{L}}$-alanine solution at room temperature took place in $163 \pm 11 \mathrm{~min}$ and $161 \pm 6 \mathrm{~min}$ on circular blank PMMA and SNFs-coated PMMA platforms, respectively. On the blank side of circular PMMA platforms, the size of ${ }_{\mathrm{L}}$-alanine crystals ranged from 251 to $732 \mu \mathrm{m}$, similar to those grown on SNFs-coated side of the circular PMMA platforms (154 to $704 \mu \mathrm{m}$, see Fig. S28, ESI $\dagger$ for crystal size distribution). Using MA-MAEC, the complete evaporation of the solvent was reduced to $49 \pm 4$ min and $42 \pm 2$ min on circular blank PMMA and SNFs-coated PMMA platforms, respectively. As compared to room temperature crystallization, the size range for L-alanine crystals grown using MA-MAEC technique further increased up to $\sim 1800 \mu \mathrm{m}$ (Table 1, SNFs Microwave and Fig. S28, ESI $\dagger$ ). Optical images (Fig. S3-S7, ESI $\dagger$ ) show that ${ }_{\mathrm{L}}$-alanine crystals were of the $\{a\}$-form. It is important to note that the complete evaporation of solvent was carried out to investigate the effect of solvent evaporation on the crystallization process. L-alanine crystals appear before the complete evaporation of the solvent and one can harvest $\mathrm{L}$-alanine crystals at any time with desired crystal size.

Since our research group has previously demonstrated the rapid crystallization of $\mathrm{L}$-alanine on blank glass slides and SNFs using MA-MAEC, ${ }^{12}$ it is important to compare those results with the ones obtained using the circular PMMA platforms in this study. One of the important differences between two platforms is the complete evaporation time for the same volume sample. On blank glass slides and SNFs-coated glass slides (no silicon isolator was used), the complete evaporation for the solvent occurred 41-50 $\mathrm{min}$ at room temperature and 6-7 min using microwave heating (at power level 1). These values are increased to 161-163 min and 42-49 min for room temperature and microwave heating (power level 1), which can be attributed to the ratio of surface area per volume of the sample. Initial $\mathrm{L}$-alanine solution $\left(20 \mu \mathrm{L}\right.$ ) spreads to an area of $\sim 400 \mathrm{~mm}^{2}$ on blank glass slides and SNFs-coated glass slides, as compared to an area of $\sim 12 \mathrm{~mm}^{2}$ on circular PMMA platforms with 16 wells, which corresponds to a $\sim 33$-fold difference in the surface area. Since silver is an excellent conductor of heat and heat transfer by conduction occurs at a faster rate on a larger area of the identical silvered surface, a $20 \mu \mathrm{l}$ solution is expected to evaporate faster on SNFs-coated glass slides than on circular PMMA platforms. The increase in size of ${ }_{\mathrm{L}}$-alanine crystals grown on circular PMMA platforms as compared to glass slides can also be attributed to differences in the ratio of surface area to sample volume. In addition, since the microwave heating of solutions on circular PMMA platforms is more homogenous than that on glass slides, the growth of ${ }_{\mathrm{L}}$-alanine crystals occurred under more temperature-controlled conditions on circular PMMA platforms. This is also partly due to the use of silicon isolators on PMMA disks, which absorb excess microwave heating. A significant amount of 
amorphous matter was previously observed on glass slides, ${ }^{12}$ where on circular PMMA platforms the formation of amorphous matter was negligible. Moreover, the extent of single L-alanine crystals was higher on circular PMMA platforms as compared to those grown on glass slides. These observations imply that circular PMMA platforms are superior to their glass slides counterparts as a rapid crystallization platform.

Subsequent to the demonstration of use of our circular PMMA platform in MA-MAECbased rapid crystallization of $\mathrm{L}_{\mathrm{L}}$-alanine without additives, the effect of two additives (L-valine

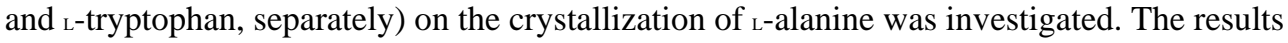

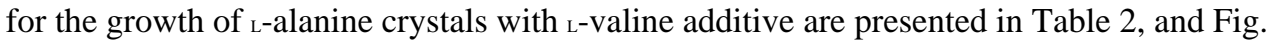
S8-S12 and S29 (ESI $\dagger$ ). At room temperature, the average complete evaporation time of $\mathrm{L}^{-}$ alanine solution with $\mathrm{L}$-valine additive both on blank and SNFs-coated sides of the circular PMMA platforms was observed to be $191 \pm 24 \mathrm{~min}$. The size of the ${ }_{\mathrm{L}}$-alanine crystals grown with ${ }_{L}$-valine additive ranged between 385-1240 $\mu \mathrm{m}$ on blank PMMA and 695-2396 $\mu \mathrm{m}$ on SNFs (see Fig. S29, ESI $\dagger$ for crystal size distribution). Using MA-MAEC technique, the complete evaporation of the identical solution occurred in $43 \pm 3$ min on blank PMMA, and

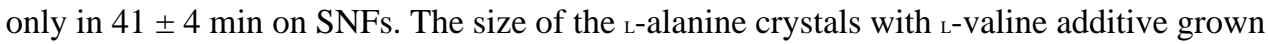
on blank PMMA ranged between 333-1124 $\mu \mathrm{m}$, while those grown on SNFs ranged between 390-1906 $\mu \mathrm{m}$ (see Fig. S29, ESI† for crystal size distribution). It is important to

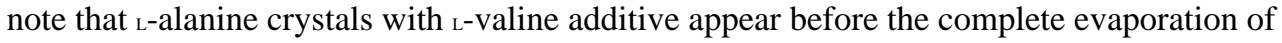

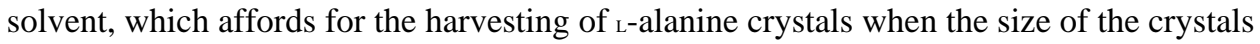
reached a desired range Fig. S8-S12, ESI $\dagger$ ). In addition, ${ }_{L}$-alanine crystals of high quality (without imperfections) were grown using MA-MAEC, particularly before the complete evaporation of the solvent. However, L-alanine crystals had more imperfections after the complete evaporation of the solvent, which implies that complete evaporation of the solvent should be avoided for the sake of growing L-alanine crystals of high quality.

Table 3 and Fig. 3 and 4, S13-S15 and S30 (ESI †) summarizes the results for the crystallization of ${ }_{\mathrm{L}}$-alanine with $\mathrm{L}$-tryptophan additive. At room temperature, the complete evaporation of solvent occurred on average in $194 \pm 9$ min and $190 \pm 11 \mathrm{~min}$ on blank and SNFs-coated sides of the circular PMMA platforms, respectively. The size of the ${ }_{\mathrm{L}}$-alanine crystals with ${ }_{L}$-tryptophan additive ranged between $257-1184 \mu \mathrm{m}$ on blank PMMA and $422-$ $2992 \mu \mathrm{m}$ on SNFs (see Fig. S30, ESI $\dagger$ for crystal size distribution). Using MA-MAEC technique, the complete evaporation of the identical solution occurred in $37 \pm 5 \mathrm{~min}$ on blank PMMA, and only in $32 \pm 7$ min on SNFs. The size of the ${ }_{L}$-alanine crystals with $\mathrm{L}^{-}$ tryptophan additive grown on blank PMMA ranged between 237-1516 $\mu \mathrm{m}$, while those grown on SNFs ranged between 327-1742 $\mu \mathrm{m}$ see Fig. S30, ESI $†$ for crystal size distribution).

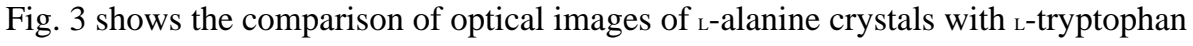
additive grown on circular PMMA platforms at room temperature and using MA-MAEC technique. After complete evaporation of the solvent, ${ }_{\text {- }}$-alanine crystals with ${ }_{\mathrm{L}}$-tryptophan additive grown on blank PMMA at room temperature appeared to have the most unorganized crystals (i.e., variable optical transparency and planar defects) with large number of polycrystals. On the other hand, L-alanine crystals with L-tryptophan additive grown on SNFs both at room temperature and using MA-MAEC were mostly single crystals

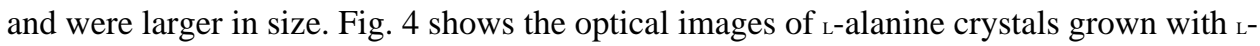
tryptophan additive during the solvent evaporation process on circular PMMA platforms

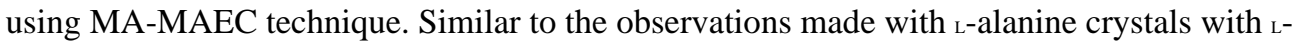
valine additive, L-alanine crystals grown with L-tryptophan additive were observed before the

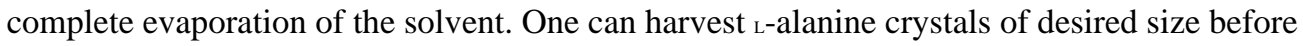
the complete evaporation of the solvent. 
The overall comparison of the optical images of L-alanine crystals grown without additives, with ${ }_{\mathrm{L}}$-valine additive and with $\mathrm{L}$-tryptophan additive on circular PMMA platforms using MA-MAEC technique is shown in Fig. 5. While all L-alanine crystals were of $\{a\}$-form, those grown by MA-MAEC differed in size and physical characteristics depending on the additive used. L-alanine crystals grown without additives were the smallest as compared to Lalanine crystals grown with both additives. In addition, $\mathrm{L}$-alanine crystals grown without additives were relatively round in shape, though several crystals with thin and elongated faces were also observed. On the other hand, $\mathrm{L}$-alanine crystals grown with $\mathrm{L}$-valine and $\mathrm{L}_{\mathrm{L}}$ tryptophan additives were mostly elongated with a lesser extent of crystals possessing a rounder shape. $\mathrm{L}$-alanine without additives and $\mathrm{L}$-alanine grown with $\mathrm{L}$-valine crystals also had some imperfections (i.e., variable optical transparency and planar defects), while $\mathrm{L}^{-}$ alanine grown with L-tryptophan crystals had significantly less imperfections. The presence of imperfections on the ${ }_{\mathrm{L}}$-alanine crystals is attributed to the spiral growth mechanism, as described elsewhere. ${ }^{22}$ In short, it was suggested that the attachment of additives to L-alanine molecules results in the disruption in the formation of the next growing layer. Thus, the layer with an additive forms an imperfection and prevents the development of a smooth, flat surface on the plane of the particular face, ${ }^{23}$ which is particularly seen in crystals grown using MA-MAEC.

L-Alanine crystals were characterized using Raman spectroscopy and powder XRD. As shown in Fig. 6 and S16-S21, ESI $\dagger$, --alanine crystals with additives displayed identical peaks as those of $\mathrm{L}$-alanine crystals grown without additives. It is important to note that the extent of both additives were $0.5 \% \mathrm{w} / \mathrm{w}$. Due to the similarities in the structures of $\mathrm{L}_{\mathrm{L}}$ alanine and $\mathrm{L}_{\mathrm{L}}$ valine molecules (L-valine has an additional isopropyl), the observation of identical Raman spectra for $\mathrm{L}_{\mathrm{L}}$-alanine without additives and $\mathrm{L}_{\mathrm{L}}$ alanine with $\mathrm{L}_{\mathrm{L}}$-valine is expected. Although L-tryptophan differs from L-alanine with an indole structure, comprised of $-\mathrm{CH},-$ $\mathrm{C}-\mathrm{C},-\mathrm{C}=\mathrm{C}-,-\mathrm{NH}$ and $-\mathrm{CN}$ bonds within the indole ring, the Raman peaks for these bonds were indistinguishable from the Raman peaks for L-alanine without additives. Further characterization of $\mathrm{L}_{\mathrm{a}}$-alanine crystals were carried out by powder XRD.

Fig. 7 and S22-S27, ESI $\dagger$ show the powder XRD for ${ }_{\mathrm{L}}$-alanine crystal grown with and without additives on circular PMMA platforms using MA-MAEC technique. All diffraction peaks for $\mathrm{L}_{\text {-alanine crystals grown with and without additives appear at the identical angles }}$ and are in good agreement with previous literature. ${ }^{24}$ For example, the (120) face is the dominant face of the ${ }_{\mathrm{L}}$-alanine crystals grown without additives. ${ }^{23}$ In this study, the (120) face is present in $\mathrm{L}_{\mathrm{L}}$-alanine crystals of all three conditions (i.e., L-alanine [no additive], $\mathrm{L}_{\mathrm{L}}$ alanine with $\mathrm{L}$-valine, and $\mathrm{L}$-alanine with $\mathrm{L}$-tryptophan). However, $\mathrm{L}$-alanine crystals grown with additives the (120) face appear to be larger in size (according to the optical images), which is thought to be due to the inhibition of the growth of other faces. Similar observations were made by other research groups for the growth of (110), (010) and (210) of L-alanine crystals without additives, which do not appear on $\mathrm{L}$-alanine crystals grown with hydrophobic $\mathrm{L}-$ leucine additive. ${ }^{23}$ Although XRD data in Fig. 7 and S22-S27, ESI $\dagger$ show that (110) and (210) faces are present on L-alanine crystals grown with additives, optical images show that these faces were not well developed. L-alanine with grown with $\mathrm{L}^{-}$ tryptophan on circular PMMA platforms are elongated and possess a wide range of size compared to pure ${ }_{\mathrm{L}}$-alanine crystals as observed by others. ${ }^{23}$ The lack of development of the (110) and (210) faces on $\mathrm{L}_{-}$-alanine crystals grown with $\mathrm{L}_{-}$-tryptophan can be attributed to selective attachment of the ${ }_{\mathrm{L}}$-tryptophan molecules onto (120) face of the ${ }_{\mathrm{L}}$-alanine crystals due to the hydrophobic interactions between $\mathrm{L}_{\mathrm{L}}$ alanine and $\mathrm{L}$-tryptophan molecules. ${ }^{23}$ Conversely, the growth of (210) face is mostly due to hydrophilic interactions between these amino acids. ${ }^{23}$ Work is currently underway for the use of circular PMMA platforms for the rapid crystallization of biological macromolecules and will be reported in due course. 


\section{Conclusions}

The use of circular PMMA platforms comprised of 16 wells in rapid crystallization of $\mathrm{L}_{\text {- }}$ alanine in the presence of additives using MA-MAEC technique was demonstrated. The circular PMMA crystallization platforms were further modified with SNFs, which served as selective nucleation sites and yielded thermal gradient when microwave heated. Theoretical simulations of heating pattern for the circular PMMA platforms in a conventional microwave oven predicted $\sim 2.5^{\circ} \mathrm{C}$ increase in temperature during microwave heating at

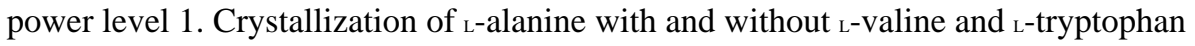
additives took 32-50 min and 161-194 min using MA-MAEC technique and room temperature evaporation respectively. Optical microscopy studies of the ${ }_{\mathrm{L}-\text { alanine crystals }}$

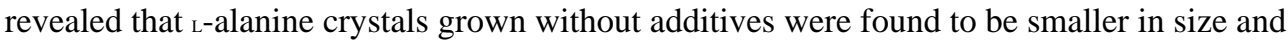
had several well-developed faces, whereas L-alanine crystals grown with additives appeared to be larger and had only dominant one highly-developed face. Raman spectroscopy and powder XRD measurements showed that all $\mathrm{L}$-alanine crystals had identical peaks, despite the morphological differences observed by optical microscope images. The results of this study imply that MA-MAEC can be carried out for multiplexed crystallization of amino acids with additives using a circular PMMA platforms with 16 wells specifically designed for MA-MAEC technique.

\section{Acknowledgments}

The project described was supported by Award Number 5-K25EB007565-05 from the National Institute of Biomedical Imaging and Bioengineering. The content is solely the responsibility of the authors and does not necessarily represent the official views of the National Institute of Biomedical Imaging and Bioengineering or the National Institutes of Health

\section{References}

1. Garetz BA, Matic J, Myerson AS. Phys. Rev. Lett. 2002; 89175501.

2. Hamilton BD, Weissbuch I, Lahav M, Hillmyer MA, Ward MD. J. Am. Chem. Soc. 2009; 131:2588-2596. [PubMed: 19053471]

3. Lee AY, Lee IS, Dettet SS, Boerner J, Myerson AS. J. Am. Chem. Soc. 2005; 127:14982-14983. [PubMed: 16248610]

4. Singh A, Myerson AS. J. Pharm. Sci. 2010; 99:3931-3940. [PubMed: 20533552]

5. Lee IS, Lee AY, Myerson AS. Pharm. Res. 2008; 25:960-968. [PubMed: 17896099]

6. Thorson MR, Goyal S, Schudel BR, Zukoski CF, Zhang GGZ, Gong Y, Kenis PJA. Lab Chip. 2011; 11:3829-3837. [PubMed: 21956673]

7. Thorson MR, Goyal S, Gong Y, Zhang GGZ, Kenis PJA. CrystEngComm. 2012; 14:2404-2412.

8. Alabanza AM, Pozharski E, Aslan K. Cryst. Growth Des. 2012; 12:346-353.

9. Pinard MA, Aslan K. Cryst. Growth Des. 2010; 10:4706-4709.

10. Cashell C, Corcoran D, Hodnett BK. Cryst. Growth Des. 2004; 5:593-597.

11. Mohammed M, Syed MF, Bhatt MJ, Hoffman EJ, Aslan K. Nano Biomed. Eng. 2012; 4:35-40. [PubMed: 22485195]

12. Alabanza AM, Aslan K. Cryst. Growth Des. 2011; 11:4300-4304.

13. Lechuga-Ballesteros D, Rodríguez-Hornedo N. Int. J. Pharm. 1995; 115:151-160.

14. Mo Y, Dang L, Wei H. Ind. Eng. Chem. Res. 2011; 50:10385-10392.

15. Poornachary SK, Chow PS, Tan RBH, Davey RJ. Cryst. Growth Des. 2007; 7:254-261.

16. Lahav M, Leiserowitz L. Chem. Eng. Sci. 2001; 56:2245-2253.

17. Lechuga-Ballesteros D, Rodriguez-Hornedo N. Pharm. Res. 1993; 10:1008-1014. [PubMed: 8378242]

18. Pinard MA, Grell TAJ, Pettis D, Mohammed M, Aslan K. CrystEngComm. 2012; 14:4557-4561. [PubMed: 22888307] 
19. Jaeblon T. J. Am. Acad. Orthop. Surg. 2010; 18:297-305. [PubMed: 20435880]

20. Gautschi OP, Schlett CL, Fournier J-Y, Cadosch D. Clin. Neurol. Neurosurg. 2010; 112:915-916. [PubMed: 20719430]

21. Aslan K, Geddes CD. Anal. Chem. 2009; 81:6913-6922. [PubMed: 19601619]

22. Burton WK, Cabrera N, Frank FC. Philos. Trans. R. Soc. London, Ser. A. 1951; 243:299-358.

23. Lechuga-Ballesteros DR-H, N. Int. J. Pharm. 1995; 115:151-160.

24. Caroline ML, Sankar R, Indirani RM, Vasudevan S. Mater. Chem. Phys. 2009; 114:490-494. 


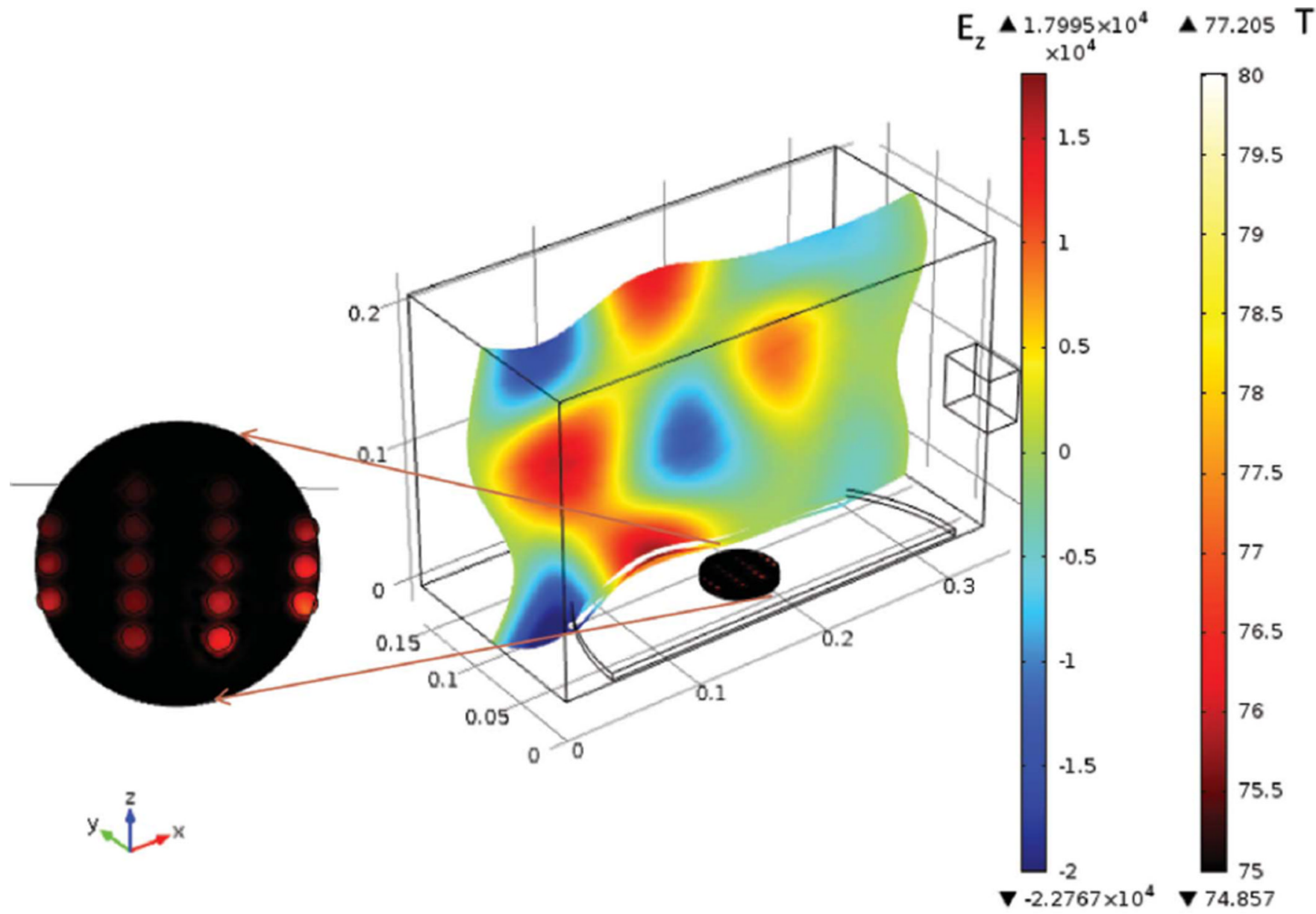

Fig. 1.

Computer simulation (using COMSOL Multiphysics ${ }^{\mathrm{TM}}$ ) of heating pattern in a conventional microwave oven after $3 \mathrm{~s}$. Temperature $(T)$ of the solution inside wells did not rise above $77.5^{\circ} \mathrm{C}$ throughout entire heating cycle. $Z$-component of the electric field $\left(E_{Z}: \mathrm{V} \mathrm{m}^{-1}\right)$ is shown. 


\section{UV-VIS Absorption Spectra of Crystallization Platforms}

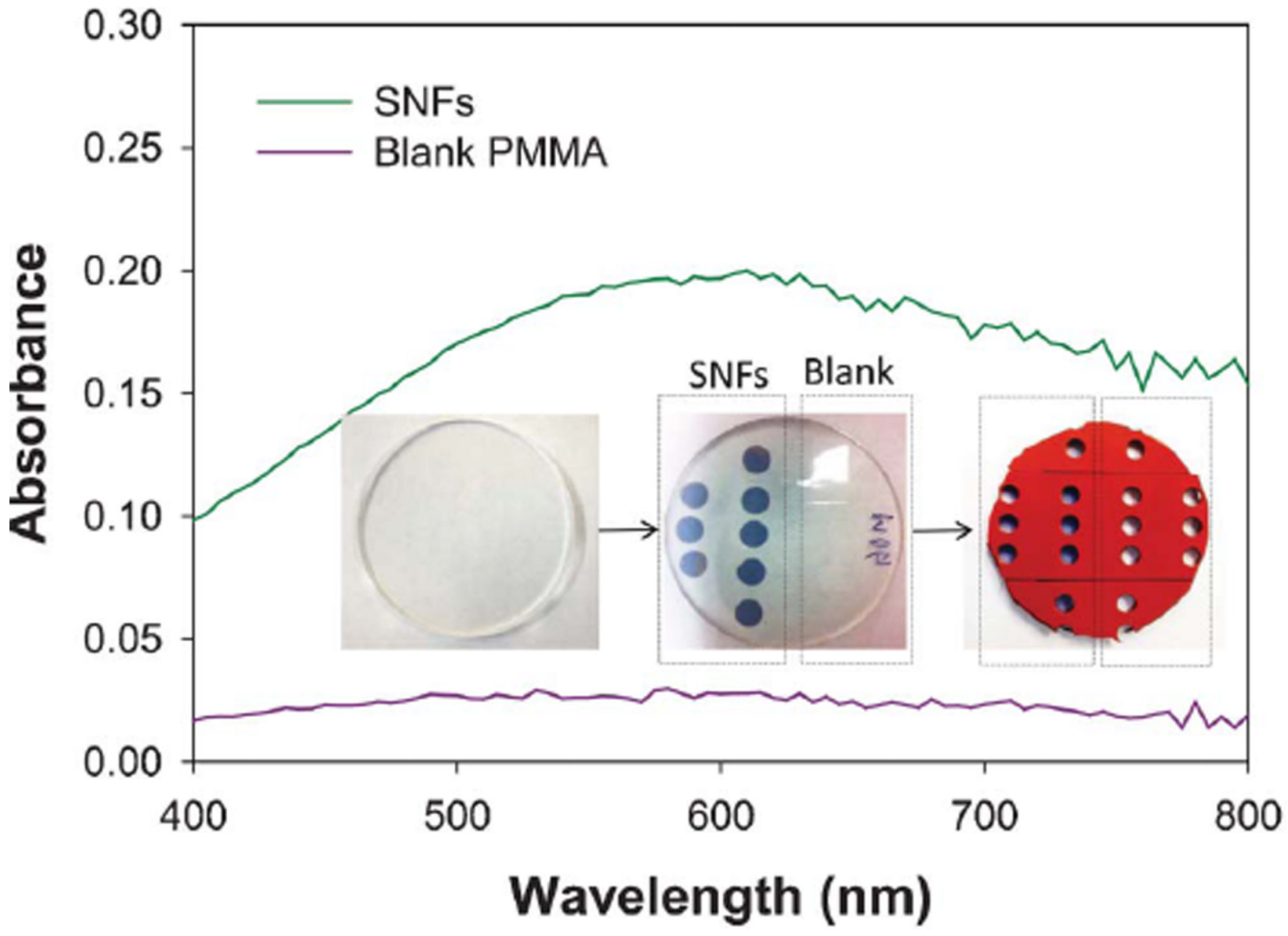

Fig. 2.

Absorbance spectra of blank PMMA disk and SNFs-coated PMMA platform. The real-color photographs show the transformation of the blank PMMA disks to SNFs-coated PMMA platforms. 


\section{L-alanine + tryptophan}
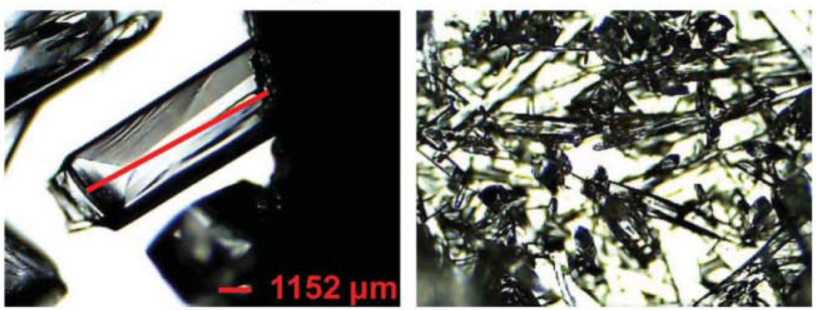

PMMA - Room Temp
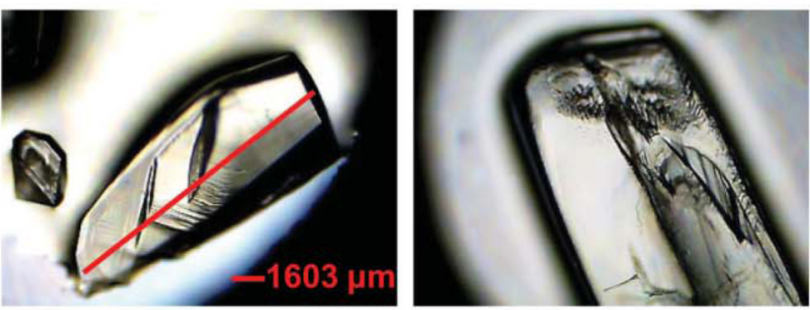

SNFs - Room Temp

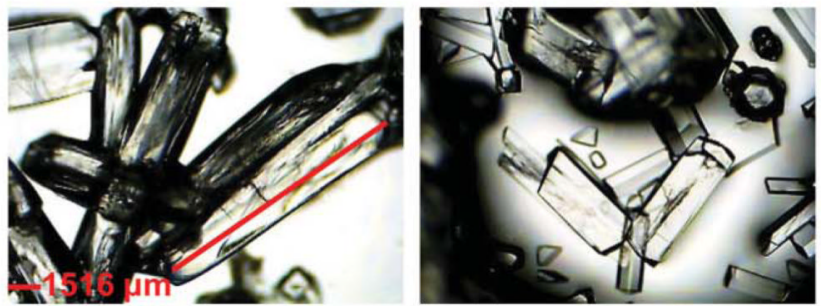

PMMA - Microwave
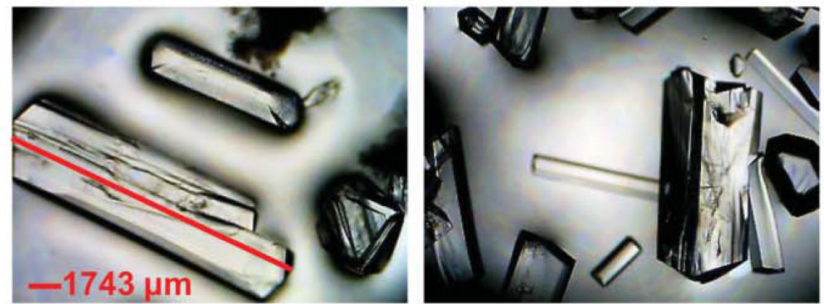

SNFs - Microwave

Fig. 3.

Optical images of crystals formed from 2.0 $\mathrm{M}_{\mathrm{L}}$-alanine solution with $\mathrm{L}$-tryptophan additive on blank PMMA disks and SNFs at room temperature and using MA-MAEC technique. 


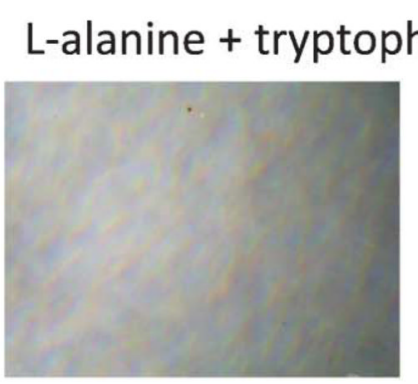

$0 \min$

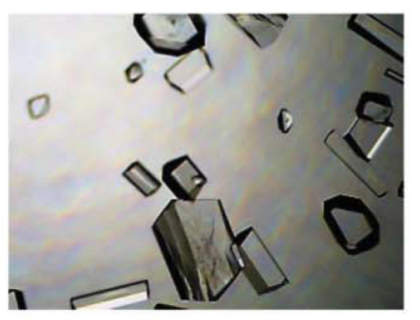

$10 \mathrm{~min}$

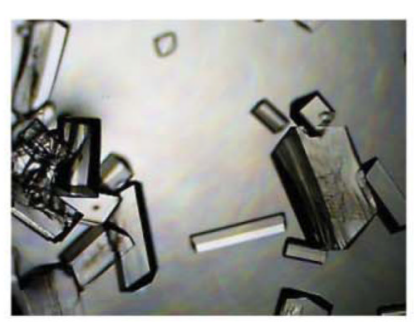

$15 \min$

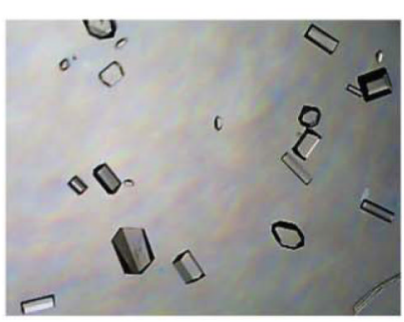

$3 \min$

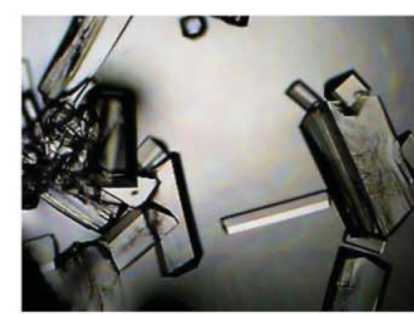

$20 \min$

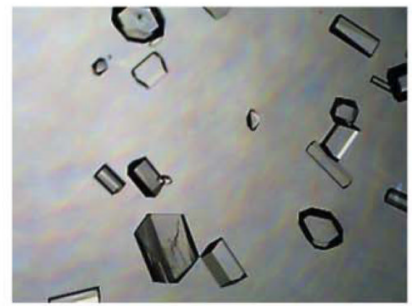

$5 \mathrm{~min}$

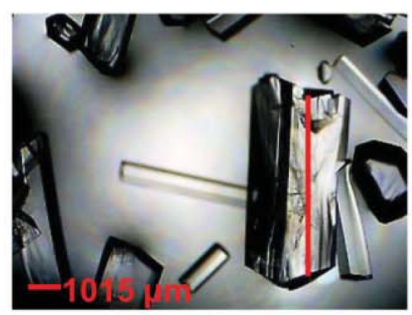

$30 \mathrm{~min}$

Fig. 4.

Time progression of the growth of ${ }_{\mathrm{L}}$-alanine crystals with $\mathrm{L}_{\mathrm{-}}$ tryptophan on SNFs using MAMAEC technique. 
L-alanine + no additive_SNFs_MW

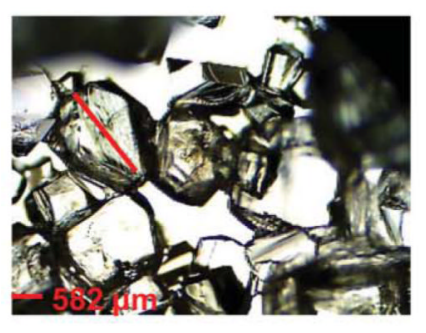

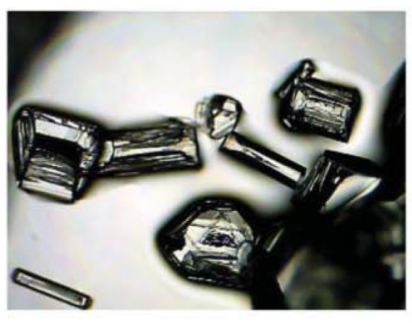

L-alanine + tryptophan_SNFs_MW

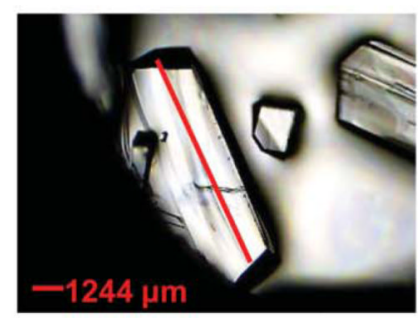

L-alanine + valine_SNFs_MW
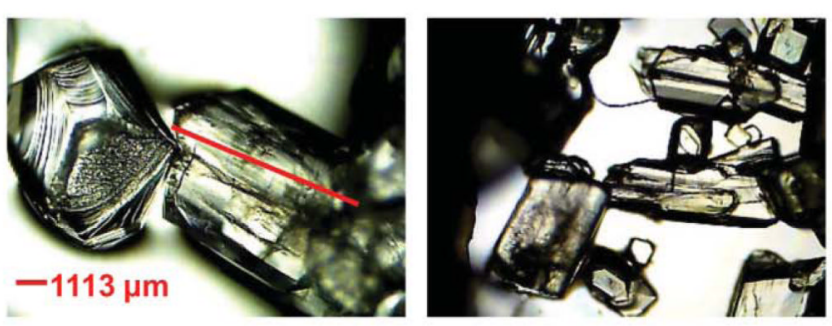

Fig. 5.

Comparison between $\mathrm{L}_{\mathrm{L}}$-alanine (no additive), $\mathrm{L}$-alanine with valine, and $\mathrm{L}$-alanine with tryptophan using MA-MAEC.

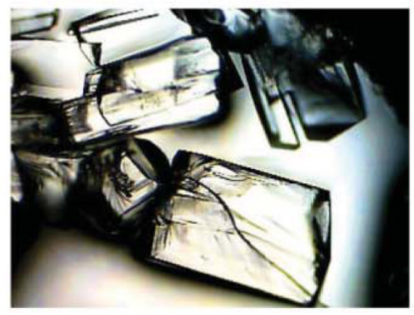




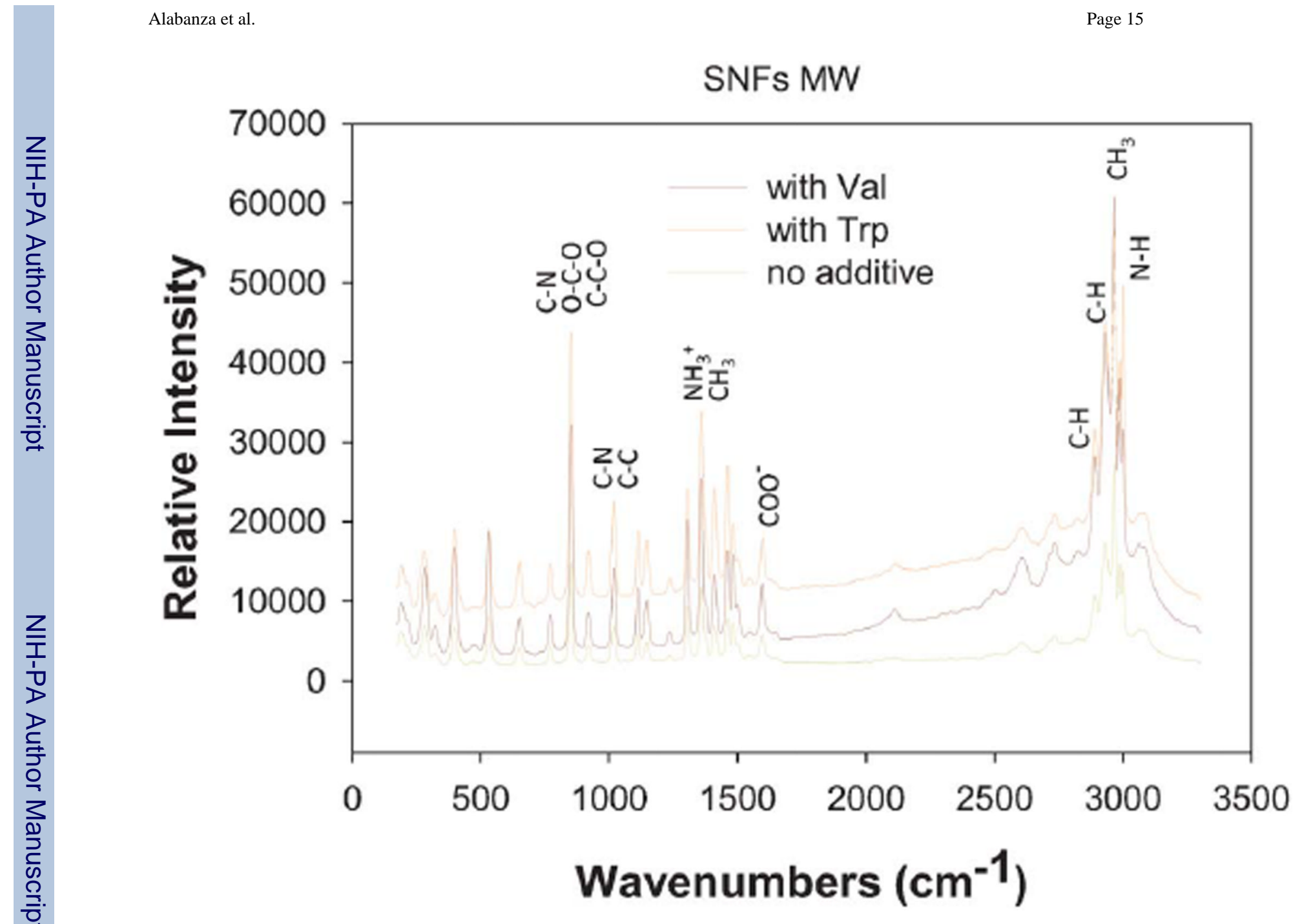

Fig. 6.

Raman spectrum of $\mathrm{L}_{\mathrm{L}}$-alanine crystals grown with no additive, $\mathrm{L}$-valine, and L-tryptophan using MA-MAEC. Functional group assignments of each significant peak are listed. 
SNFs MW

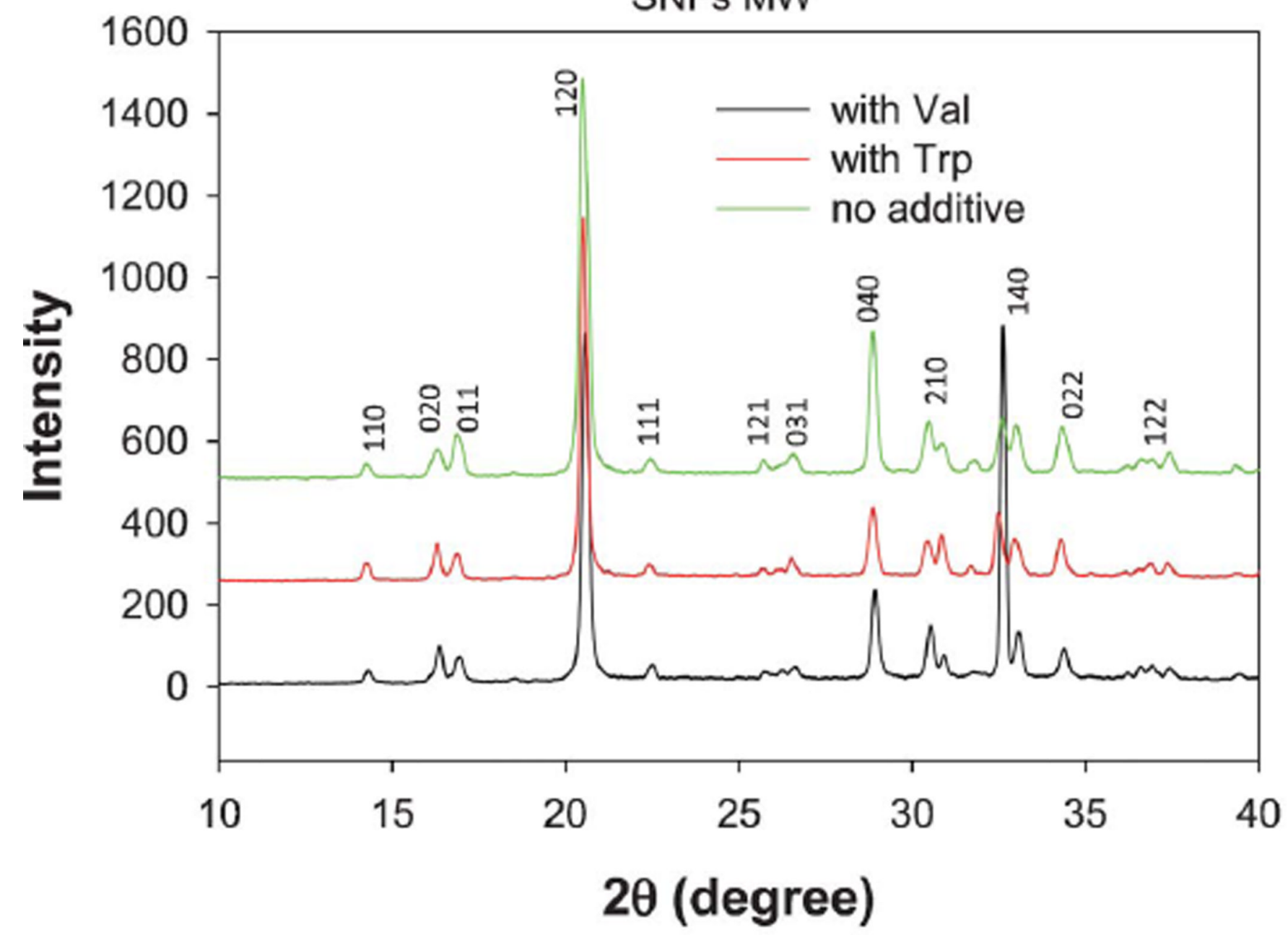

Fig. 7.

Power X-ray diffraction (XRD) measurements of $\mathrm{L}_{\mathrm{-}}$ alanine crystals with no additive, $\mathrm{L}_{\text {- }}$ valine, and $\mathrm{L}$-tryptophan. Corresponding faces are labeled above peaks. 


\section{Table 1}

Summary of results for the crystallization of ${ }_{\mathrm{L}}$-alanine from 2.0 M solution on blank PMMA disks and silver nanostructured films (SNFs). Crystallization was performed at room temperature and using MA-MAEC technique. $N=5$ samples

\begin{tabular}{lll}
\hline L-Alanine (no additive) & Crystallization time & Size range $(\boldsymbol{\mu m})$ \\
\hline PMMA room temperature & $163 \pm 11 \mathrm{~min}$ & $251-732$ \\
SNFs room temperature & $161 \pm 6 \mathrm{~min}$ & $154-704$ \\
PMMA microwave & $49 \pm 4 \mathrm{~min}$ & $240-889$ \\
SNFs microwave & $42 \pm 2 \mathrm{~min}$ & $251-1774$ \\
\hline
\end{tabular}




\section{Table 2}

Summary of results for the crystallization of ${ }_{\mathrm{L}}$-alanine from $2.0 \mathrm{M}$ solution with $0.5 \%(\mathrm{w} / \mathrm{w})_{\mathrm{L}}$-valine additive on blank PMMA disks and SNFs. Crystallization was performed at room temperature and using MA-MAEC technique. $N=5$ samples

\begin{tabular}{lll}
\hline L-Alanine + L-valine & Crystallization time & Size range $(\boldsymbol{\mu m})$ \\
\hline PMMA room temperature & $191 \pm 24 \mathrm{~min}$ & $385-1240$ \\
SNFs room temperature & $191 \pm 16 \mathrm{~min}$ & $695-2396$ \\
PMMA microwave & $43 \pm 3 \mathrm{~min}$ & $333-1124$ \\
SNFs microwave & $41 \pm 4 \mathrm{~min}$ & $390-1906$ \\
\hline
\end{tabular}




\section{Table 3}

Summary of results for the crystallization of ${ }_{\mathrm{L}}$-alanine from $2.0 \mathrm{M}$ solution with $0.5 \%(\mathrm{w} / \mathrm{w})_{\mathrm{L}}$-tryptophan additive on blank PMMA disks and SNFs. Crystallization was performed at room temperature and using MAMAEC technique. $N=5$ samples

\begin{tabular}{lll}
\hline L-Alanine + L-tryptophan & Crystallization time & Size range $(\boldsymbol{\mu m})$ \\
\hline PMMA room temperature & $194 \pm 9 \mathrm{~min}$ & $257-1184$ \\
SNFs room temperature & $190 \pm 11 \mathrm{~min}$ & $422-2992$ \\
PMMA microwave & $37 \pm 5 \mathrm{~min}$ & $237-1516$ \\
SNFs microwave & $32 \pm 7 \mathrm{~min}$ & $327-1742$ \\
\hline
\end{tabular}

